\title{
Estrategia e intervención estatal en la siderúrgica colombiana: consideraciones desde el institucionalismo
}

Strategy and state intervention in the colombian steel mill: considerations from institutionalism

Stratégie e intervention de l'état dans la sidérurgie colombienne: considérations du point de vue de l'institutionnalisme

Patricia Carolina Barreto Bernal patinpe@gmail.com Avenida central del Norte, UPTC, edificio central oficina 343, Tunja.

Estudiante del Doctorado en Administración de la Universidad EAFIT. Magister en Administración del Instituto

Tecnológico de Estudios

Superiores de Monterrey y

la Universidad Autónoma de Bucaramanga. Profesora Asistente de la Universidad Pedagógica y Tecnológica de Colombia (UPTC) Tunja, Facultad de Ciencias

Económicas y Administrativas,

Escuela de Administración de

Empresas. Grado de Honor como Administradora de Empresas UPTC.

Artículo de investigación científica y tecnológica Según Clasificación Colciencias

Fecha de recepción: septiembre 03 de 2011 Fecha de corrección: octubre 13 de 2011 Fecha de aprobación: diciembre 20 de 2011 


\section{Strategy and state intervention in the colombian steel mill: considerations from institutionalism}

\section{Estrategia e intervención estatal en la siderúrgica colombiana: consideraciones desde el institucionalismo}

\section{Stratégie e intervention de l'état dans la sidérurgie colombienne: considérations du point de vue de l'institutionnalisme}

\section{Stratégie e intervention de l'état dans la sidérurgie colombienne: considérations du point de vue de l'institutionnalisme}

\author{
Estrategia e intervención estatal en la \\ siderúrgica colombiana: consideraciones \\ desde el institucionalismo
}

\author{
Strategy and state intervention in the \\ colombian steel mill: considerations from \\ institutionalism
}

\section{Résumée}

Le secteur sidérurgique présente dans ses origines et son développement historique, les caractéristiques typiques d'un comportement d'isomorphisme dans le cadre d'analyse institutionnel qui met en évidence deux réalités du secteur. D'une part, le changement du rôle de l'État, qui a passé de promoteur et contrôleur direct à médiateur des conditions de compétitivité des entreprises sidérurgiques face au marché international. D’autre part, l'analyse des conditions institutionnalisées du secteur sidérurgique permet observer que les possibilités de décisions stratégiques conçues par les entreprises sont limitées, face à la complexité des pressions externes. Ceci met l'accent sur le degré d’isomorphisme, qui passe d'une approche régulatrice à une approche normative et cognitive. Si bien que quelques tendances du changement institutionnalisé du secteur à niveau mondial sont présentes, on approfondi sur la situation du secteur sidérurgique colombien : dès ses origines avec les entreprises dédiées à l'exploitation du fer au XIX siècle, jusqu'à son actuelle composition de fusions et d'acquisitions avec l'investissement étranger des groupes Votorantim et Gerdau du Brésil. Cette réflexion a pour objectif de montrer les possibilités offertes par l'approche institutionnelle pour analyser le changement stratégique de l'évolution historique d'un secteur industriel.

Mots clef: institutionnalisme, sidérurgique, isomorphisme, changement, stratégie, normes, gouvernement. 


\section{Estrategia e intervención estatal en la siderúrgica colombiana: consideraciones desde el institucionalismo}

\section{Introducción}

La dinámica de desarrollo de los países industrializados tuvo en sus orígenes estrechas relaciones con el establecimiento del sector siderúrgico, subsidiado por el Estado desde antes de la revolución industrial. Pero, ¿Qué ha pasado ahora con esta industria en el entorno competitivo de la economía globalizada?, ¿Sigue siendo la industria más protegida de los gobiernos?, ¿Cómo se configuró la tendencia estratégica de estas industrias a lo largo del siglo XX? Estas preguntas invitan a dar un vistazo sobre la evolución histórica del sector siderúrgico en Colombia, lo cual constituye uno de los propósitos de este trabajo.

La extracción de minerales como carbón, hierro y caliza, constituye un factor de vital importancia en la producción siderúrgica integrada, sin embargo, la estructura logística requerida para transformar los minerales en productos de hierro y acero es de grandes dimensiones y necesita altas inversiones de capital tanto para la tecnología de producción como para la logística de entrada de materias primas y salida de productos terminados.

Por lo anterior, la participación del Estado en la consolidación y protección de este sector se ha hecho explícita, primero, en la colocación directa de recursos públicos para inversión en este tipo de empresas, segundo, con la creación de normas que fomentan el mercado interno de los países, dinamizando el sector de la construcción y la creación de nuevos renglones industriales para la estimulación de la demanda de productos siderúrgicos y tercero, con la creación de medidas de protección frente a los mercados externos.

Sin embargo, en el transcurso de la segunda mitad siglo $X X$, la apertura de los mercados mundiales y la creación de bloques económicos, impuso una nueva dinámica de los mercados industriales donde el sector siderúrgico no fue la excepción. Se agudizaron las condiciones de competencia entre las empresas siderúrgicas más importantes del mundo, las cuales fueron ganando cada vez más capacidad de producción y, por lo tanto, mejores condiciones para competir por los segmentos del mercado mundial, lo cual ha implicado, paralelamente, un cambio en la relación Estado - empresas siderúrgicas, que en Colombia no es del todo clara.

Si bien es cierto, la apertura de mercado impone mayores retos en las estrategias competitivas de los sectores industriales, también es indudable que en ciertas industrias pesadas, como la siderúrgica, las condiciones de infraestructura y de regulación estatal son indispensables para apoyar un crecimiento sostenido que pueda desarrollar capacidades de respuesta estratégicas frente a las presiones competitivas del entorno.

El artículo presenta una mirada a la evolución histórica del papel del Estado frente a las condiciones de creación y evolución del sector siderúrgico en Colombia. Allí, la teoría institucional brinda elementos pertinentes para establecer las relaciones entre las medidas de regulación gubernamental, la normatividad técnica y las respuestas estratégicas adoptadas por las empresas siderúrgicas en el marco de la institucionalización de ciertas prácticas industriales específicas.

El texto se conforma en tres partes, inicialmente se plantean los elementos básicos de la teoría institucional que constituyen el marco de análisis para abordar el estudio de un sector industrial, y los mecanismos o posibles respuestas estratégicas que podrían adoptar las empresas que lo conforman cuando dependen de un alto grado de proteccionismo.

Luego, se presenta la discusión para el caso de la industria siderúrgica colombiana, en un breve recorrido sobre el papel que el Estado ha desarrollado en las diferentes fases de evolución del sector y las respuestas estratégicas que el empresariado a dado a los cambios en las dinámicas económica y comercial en ésta industria. Finalmente se presentan algunas reflexiones a manera de conclusiones, que abren preguntas para nuevas investigaciones sobre el futuro del 
sector aún dentro de un esquema globalizado y competitivo a nivel mundial.

\section{Supuestos teóricos que aporta el institucionalismo}

Se reconocen dos corrientes de pensamiento distintas dentro del institucionalismo, la económica y la sociológica. En ambos enfoques se encuentran elementos para analizar el ambiente competitivo de los sectores económicos.

Desde la mirada económica, el institucionalismo permite analizar el impacto de las transacciones entre los agentes y las relaciones interinstitucionales, poniendo el énfasis en los costos de transacción, los acuerdos contractuales y los resultados económicos de las firmas.

Dentro de este enfoque se destaca el aporte de Douglas North, quien define elementos importantes para comprender la dinámica formal de las interacciones entre instituciones, y quien define las normas como:

Las reglas formales incluyen reglas políticas (y judiciales), reglas económicas y contratos. La jerarquía de tales reglas, desde constituciones, estatutos y leyes comunes hasta disposiciones especiales, y finalmente a contratos individuales, define limitaciones que pueden fluctuar de reglas generales a especificaciones particulares. Las reglas específicas definen derechos de propiedad, es decir el conjunto de derechos sobre el uso y el ingreso que se derivan de la propiedad y la capacidad para enajenar un valor o un recurso. La función de las normas es facilitar el intercambio político o económico (North, 1993, p.67-68)

Desde este enfoque, el centro de las relaciones entre organizaciones son las normas y las reglas de juego claras, que van a legitimar el comportamiento de los agentes en el mercado y que institucionalizan procedimientos y acciones empresariales en un mercado específico.

En cambio, desde la postura sociológica institucional, se reconocen actores sociales que legitiman las normas que van a regular los intercambios, responsabilidades y roles de los actores socialmente reconocidos.

La teoría institucional examina el papel de la influencia social y las presiones para la conformidad social en la conformación de las acciones de las organizaciones. Basándose en una perspectiva institucional, este trabajo sostiene que la selección de recursos y una ventaja competitiva sostenible están profundamente influenciados, en el individuo, la empresa y el nivel entre empresas, por el contexto institucional de las decisiones de los recursos. El contexto institucional se refiere aquí a las reglas, normas y creencias que rodean la actividad económica que definen o hacen cumplir el comportamiento económico y socialmente aceptable (Oliver, 2008, p.498)

Esta perspectiva asume una posición fuertemente determinista frente a la acción de los actores estratégicos, aunque como veremos más adelante, sutilmente les deja un campo de acción que se puede abordar desde el microinstitucionalismo.

Sin embargo, en cualquiera de los dos enfoques, la corriente institucionalista tiene su eje en la presión inexorable del entorno sobre las organizaciones, como aclaran Pffefer y Salancik a continuación: "La perspectiva niega la validez de la conceptualización de las organizaciones como la propia dirección, actores autónomos que persiguen sus propios fines y en su lugar afirma que las organizaciones son dirigidas por otros, participan en una lucha constante por la autonomía y discreción, frente a la coacción y el control externo" (1978, p.260).

Es decir, que la perspectiva institucionalista se identifica como determinística, pues preestablece el marco dentro del cual las organizaciones actúan; las reglas que van a mediar el intercambio transaccional establecen los beneficios o sanciones según la adopción o incumplimiento de las mismas.

Sin embargo, autores como Cristine Oliver, que se identifican con la teoría sociológica institucional, presentan algunos análisis en los cuales se dan miradas menos determi- 
nantes sobre la influencia del entorno en las organizaciones y abre el espacio a la posibilidad de respuesta de los empresarios a través de los juegos de poder en los escenarios en los que se determinan las normas que van a regular el intercambio comercial.

\subsection{El término institucionalidad}

Según Richard Scott, "el marco institucional define el fin y crea el significado por el cual los intereses de los diferentes actores son determinados y perseguidos. Los factores institucionales determinan quienes son los actores en un tipo de escenario. Las llamadas firmas persiguen beneficios, y en otro escenario son llamadas agencias y buscan grandes presupuestos" (1987 p 508)

Siguiendo a Scott, para el presente análisis, la institucionalidad se va a referir no a un tipo de organización específica sino al grado de incorporación de las normas, reglas y buenos procedimientos de las organizaciones que hayan sido asumidos como propios a través del tiempo, legitimados por la sociedad y las empresas del sector siderúrgico como tal.

Para los autores institucionales el cambio organizacional es el producto del cambio en el entorno pero en la medida en que se practican de manera continua ciertos comportamientos y se van adhiriendo con el tiempo a las costumbres e identidad de una organización, dichos comportamientos se transforman en rutinas, tradiciones y hábitos que socialmente son legitimados por el conjunto de actores sociales que los identifican como socialmente correctos y aceptados logrando la institucionalización de dicha conducta.

Según Hoffman (2001) esto se explica porque las fuerzas sociales definen la aceptación de prácticas y orientan a las empresas a adoptarlas. "La influencia de estas fuerzas es en términos generales de la industria y no para una firma específica,... Al final las firmas son un reflejo de la sociedad de la cual hacen parte, no directamente, pero como mediadoras y filtradoras de los ambientes institucionales".

\subsection{El isomorfismo organizacional}

El nuevo institucionalismo presenta en detalle para el análisis organizacional los efectos del cambio institucional en donde se identifica la homogenización como uno de los más evidentes y paradójicos en relación a las teorías de la estrategia, pues en esta perspectiva no se analizan los componentes diferenciadores entre una empresa y otras sino las causas que generan la similitud entre las organizaciones.

La homogenización es identificada como un fenómeno de isomorfismo institucional y se diferencian tres tipos:

1) Isomorfismo coercitivo que se deriva de la influencia política y el problema de la legitimidad, 2) Isomorfismo mimético que resulta de normas o estándares de procedimiento como respuestas a la incertidumbre, y 3) Isomorfismo normativo, asociado con la profesionalización. (Powell y DiMaggio, 2001, p.110)

Particularmente, para el análisis del sector siderúrgico, es de particular interés profundizar en las explicaciones del isomorfismo coercitivo, dado que este es explicado en palabras de Powel y DiMaggio de la siguiente manera: "El isomorfismo coercitivo resulta de presiones tanto formales como informales que sobre unas organizaciones ejercen otras que dependen y que ejercen también las expectativas culturales en la sociedad dentro de la cual funcionan las organizaciones. Esas presiones se pueden sentir como una fuerza de persuasión o invitación a coludirse."(2001, p.110)

Como lo expone la autora Cristine Oliver, el hecho de que en las empresas deban acogerse a normas regulatorias generales, conduce a cierta homogenización de los comportamientos empresariales: "La premisa básica de la teoría institucional es, entonces, que las empresas tienden hacia la conformidad con las normas predominantes, las tradiciones y las influencias sociales en sus entornos internos y externos, conduciendo a la homogeneidad entre las empresas en sus estructuras y actividades, y que las empresas exitosas son aquellas que ganan apoyo y la legitimidad, ajustándose a las presiones sociales" (1997)

Desde esta perspectiva de análisis se puede identificar una cierta tendencia de isomorfismo en la industria siderúrgica, caracterizada comúnmente a nivel mundial por la intervención del Estado como agente 
creador, de fomento, regulador y protector de esta actividad económica y, en consecuencia, se identifican comportamientos homogéneos en las actividades de las empresas siderúrgicas que determinan un tipo especifico de orientación estratégica, dependiente en alto grado de las condiciones de los factores tecnológicos y de la posesión de los recursos primarios para la producción.

\subsection{La intervención del Estado}

Las presiones del cambio institucional que generan comportamientos isomorfos en las organizaciones provienen de tres fuentes identificadas por Powell y DiMaggio como: presiones competitivas, el Estado y las profesiones.

En el plano interinstitucional, las presiones emergentes del gobierno, las alianzas de la industria y las expectativas sociales (reglas, normas y estándares sobre la calidad del producto, la seguridad o la gestión del medio ambiente, por ejemplo) definen conductas de las empresas socialmente aceptables, y las presiones sociales comunes a todas las empresas en el sector de una misma causa para exponer las estructuras y actividades similares (DiMaggio y Powell, 1983)

Por las características del sector siderúrgico, tales como, alta inversión de capital; dependencia de los recursos naturales que constituyen su materia prima o, en el caso de las siderúrgicas semi-integradas, de la existencia de chatarra para la producción del acero; los altos costos del cambio tecnológico; la intensa competencia mundial; nos concentraremos en las presiones del Estado como fuentes del cambio isomorfo coercitivo.

En países como España, por ejemplo, la realeza y el gobierno político, han estado siempre en medio de la regulación de los factores de producción del sector siderúrgico, con la existencia de protecciones normativas sobre la circulación y propiedad de las materias primas necesarias para la producción del hierro y luego del acero.

Por su parte, en Brasil, la industria siderúrgica es uno de los ejes claves del desarrollo industrial y minero, y existe una apuesta nacional para favorecer la expansión, crecimiento y liderazgo del sector en el escenario internacional.

De igual manera, es pertinente recurrir a uno de los criterios del comportamiento institucional de las industrias identificados por Richard Scott, otro reconocido autor del institucionalismo, quien define que las variaciones en el énfasis institucional de los diversos sectores, se da a nivel de los marcos: regulatorio, normativo o cognitivo, definidos por Scott así:

- El regulativo: su base de conformidad es la conveniencia, su mecanismo es coercitivo, su lógica es instrumental, sus indicadores son la reglas, leyes y sanciones, y sus bases de legitimidad son las sanciones legales.

- Normativo: La base de su conformidad son las Obligaciones sociales, su mecanismo es normativo, su lógica es la adecuación, su indicador es la certificación y la acreditación, su base de legitimidad es el gobierno moral. Los sistemas normativos incluyen ambos, valores y normas.

- Cognoscitivo: la base de conformidad es la toma de garantías, el mecanismo es mimético, su lógica es ortodoxa, los indicadores son prevalencia e isomorfismo, y su legitimidad son bases culturales y conceptualmente correctas. (Scott, 1995)

La industria siderúrgica, ha presentado a lo largo de su desarrollo énfasis en comportamientos institucionalizados, primero desde las reglas y decretos, luego, desde la apertura se han implementado más normas de estandarización nacionales e internacionales $\mathrm{y}$, últimamente, se han visto más comportamientos instituidos por iniciativa de las empresas con relación a buenas prácticas ambientales y de responsabilidad social empresariales que son legitimadas por los stakeholders del sector.

En Cuadro 1 se presenta un panorama de los principales cambios que han ocurrido en diferentes periodos sobre la intervención del Estado en el sector siderúrgico y se puede observar como ha pasado de un énfasis regulatorio a un énfasis normativo. Sin embargo, más adelante se explicará con más detalle en que consistieron las apuestas del Estado para la conformación del sector en el caso de la industria siderúrgica colombiana. 


\section{$1840-1880$}

El pais requiere una industria doméstica que le provea los materiales necesarios para la construcción de las vías ferreas y los proyectos de industriualización.

El Estado crea unas e invierte en otras Ferrerias a nivel nacional. Existieron 4 en el siglo XIX:

- Amaga en Antioquia - Pacho y la Pradera en Cundinamarca

- Samacá en Boyacá

\section{$1938-1970$}

Ley 97 de 1938, Autorizó al gobierno a crear $y$ financiar empresas siderúrgicas.

El gobierno tiene como proyecto nacional la industrialización. Se requieren productos nacionales derivados del sector siderurgico y metalmecánico que sustituyan las importaciones y satisfagan las necesidades para los proyectos de urbanización del país.

Se desarrollan las misiones geológicas para ubicar cientificamente las minas de hierro y minerales complemementarios

El Estado crea la siderurgica Nacional Acerias Paz del Río (APR) y los decretos que estimulan el mercado industrial y el de la construcción.

\section{1 - 1990}

$1991-2005$

Se da el surgimiento de diferentes iniciativas de inversion privada en el sector siderurgico.

Se avanza en los procesos competitivos de renovación y tecnificación de la industria . Aparecen las empresas con procesos de producción semi integrados. La unica integrada sigue siendo APR.

1987 CONPES para la expanción de la industria siderúrgica.

1971, Plan Indicativo de la Industria Siderúrgica.

El papel del Estado es de regulador y estimulador del mercado interno.
Participación de Colombia en el Pacto Andino.

Fusiones y adquisiciones realizadas por empresas multinacionales.

El papel del Estado es mediador desde la formulación de politicas de comercio exterior y mejoramiento de las condiciones de infraestructura para la competitivida
Con relación al cuadro se observa que la intervención del Estado en la evolución del sector inició dentro del pilar regulatorio, y que ha venido avanzando a un esquema más normativo, pero que aún no se llega a un nivel cognitivo dadas las presiones competitivas del entorno que han llevado a una respuesta estratégica menos pasiva o dependiente y más competitiva, caracterizada por las fusiones y adquisiciones o alianzas con capitales extranjeros.

Hasta aquí se tomaron en cuenta los elementos de análisis tomados desde la perspectiva institucional que servirían de apoyo para abordar el estudio de un sector económico. A continuación se enunciarán los aportes teóricos de la estrategia competitiva para el análisis de sectores industriales.

\section{El tema estratégico desde el análisis institucional}

Para analizar el comportamiento estratégico del sector siderúrgico dentro del marco conceptual de la teoría institucional es necesario tener claridad sobre las limitaciones, restricciones y posibilidades que, conceptualmente, esta corriente teórica da a la dimensión estratégica.

En este sentido, para la teoría institucional, el campo de la acción estratégica en el cambio de las condiciones del entorno, que podrían dar paso a la autonomía de la dirección empresarial, es reconocido tímidamente en el proceso institucional interno, es decir, dentro de la dinámica de las organizaciones, como plantean los autores del nuevo institucionalismo parta el análisis organizacional: 
En otras palabras, la variación en la respuesta estratégica al mismo ambiente puede producir una diferenciación en vez de isomorfismo. Especificar las condiciones en las cuales ocurre cualquiera de estos requiere prestar atención al proceso institucional interno. El microinstitucionalismo, ofrece una alternativa potente, pero aún no desarrollada, a los fuertes planteamientos de transacción costo y de la teoría de la agencia que prefieren los economistas" (Lynne G. Zucker.1991. En Powell y DiMaggio, 1999, p.152)

Adicionalmente, según Oliver, básicamente, la previsibilidad de las respuestas estratégicas organizacionales varía en función del "grado de influencia o el dominio atribuido a las organizaciones en la manipulación o el control del medio ambiente en el que se encuentran, tiene implicaciones para la presunta utilidad de la conformidad de la organización con el medio ambiente".(2008, p.501) En este sentido, la acción estratégica de las organizaciones comenzaría en la misma evaluación de la conveniencia o no de seguir, adoptar o cumplir las reglas y normas impuestas institucionalmente en un sector determinado, en contraste con los intereses particulares de la organización.

Las respuestas estratégicas previsibles de conformidad a las presiones institucionales del entorno identificadas por Oliver son: la aquiescencia, el compromiso, la evitación, el desafío y la manipulación. Estas diferencias de comportamiento organizacional frente al entorno, son un referente sustancial para el análisis de las estrategias adoptadas por las empresas en sectores industriales altamente institucionalizados como el siderúrgico.

Al respecto, podría decirse que en el sector siderúrgico, dadas las exigentes condiciones de infraestructura necesarias para desarrollar capacidades estratégicas competitivas de las organizaciones y de la alta dependencia de los beneficios estatales, ha pasado por periodos de aquiescencia y conformidad.

Según Oliver: la aquiescencia lleva a la imitación de comportamientos industriales similares y es consistente con el isomorfismo mimético planteado por Powell y DiMaggio, relacionado con la imitación consciente $o$ inconsciente de modelos institucionales y conllevan a predecir un comportamiento más orientado a la conformidad, lo cual refleja desde la teoría la realidad del sector siderúrgico en cualquiera de los escenarios mundiales en los que se pueda analizar.
Powell y DiMaggio sostienen la hipótesis que la conformidad o isomorfismo con el entorno institucional está en función de la dependencia externa. En el sector siderúrgico podríamos decir, que la mayor dependencia se da en la necesidad de intervención del Estado para procurar buenas condiciones de infraestructura, precios y aranceles frente al mercado internacional, de lo contrario, se generan la compra o fusión de las empresas ante la presión de capitales externos que tienen mayor capacidad de negociación como es el caso de Brasil, frente a las empresas siderúrgicas colombianas, dado que Brasil sí cuenta con un sólido apoyo estatal en términos de infraestructura, estímulo para la inversión y para las exportaciones en dicho sector.

Así mismo, Pfeffer y Salancik demostraron en diferentes estudios, que el cumplimiento de los programas de acción con las agencias federales y la preocupación por satisfacer las expectativas gubernamentales, se explicaban por el grado de dependencia de una organización con el gobierno.

Finalmente, Cristine Oliver describe con la siguiente cita, porqué existe un alto grado de relación entre la dependencia de los componentes institucionales que influyen en un grupo de organizaciones y la posibilidad de una respuesta de conformidad:

La conformidad parcial (es decir, de compromiso) es también una respuesta común cuando la dependencia es alta, porque las organizaciones suelen tener intereses que desean proteger o fomentar la dependencia $y$ es raramente unidireccional. Incluso en entornos institucionales muy reglamentados, las organizaciones pueden negociar con las comisiones de regulación en los términos de cumplimiento, podrán suministrar su propio personal para las agencias reguladoras como asesores, y pueden aprovechar el hecho de que la cooperación de las organizaciones en una industria es necesaria para que las comisiones hagan su trabajo (Pfeffer \& Salancik, 1978: 211). Como la dependencia de las fuentes de la disminución de la presión institucional, la prevención se convierte en una alternativa estratégica más viable (En Oliver, 2008, p.509)

En resumen, el asunto estratégico para el institucionalismo, se basa en el conocimiento profundo del sector y de las dinámicas que pueden cambiar las condiciones competitivas de los actores que participan en él. Por lo cual, las alianzas y acciones que 
desarrollen los grupos estratégicos del sector van a ser fundamentales en la determinación de las reglas de juego que se irán institucionalizando como parte de las características del sector industrial.

En este panorama el estratega no tendrá total autonomía para decidir sus movimientos de posicionamiento en el sector, sino será la dinámica del conjunto de acciones de los grupos estratégicos en la participación de los espacios posibles de intervención y proposición de nuevas reglas de juego y creación de marcos normativos convenientes para mejorar la competitividad del sector, lo que determinará los parámetros de decisión individual para lograr obtener una ventaja.

De esta manera se dejan planteados los principales aportes teóricos que el institucionalismo aporta para entrar a analizar los datos encontrados sobre la evolución de la industria siderúrgica, como un ejemplo de sector institucionalizado. Se presenta a continuación la discusión sobre el caso del sector siderúrgico en Colombia.

\section{El comportamiento del sector siderúrgico en Colombia: una mirada de su composición y cambios estratégicos en el tiempo}

El siglo XX inicia con una fuerte relación entre la evolución industrial y la política económica. Luis Ospina Vásquez en su libro Industria y Protección en Colombia entre 1810-1930, describe el empeñó del Gobierno Nacional en promover la creación de algunas industrias específicas que pudieran jalonar sectores económicos más amplios, entre esas industrias se encontraban la textil, la industria alimenticia, energética, el transporte, y la industria siderúrgica, entre otras.

Adicionalmente, el investigador Gabriel Poveda Ramos, en un informe de investigación que escribió para COLCIENCIAS, titulado "Políticas económicas, desarrollo industrial y tecnología en Colombia entre 1925 y 1975", analiza un listado de 25 medidas de política económica que contribuyeron a determinar el patrón de industrialización y desarrollo tecnológico en el país en ese período. Por considerarlas muy pertinentes para reflejar la dinámica en materia de política económica que vivía el país en la primera mitad del siglo XX, se mencionan las siguientes:
La Misión Kemmerer en 1923: por la cual se crea el Banco de la República, indispensable para la regulación financiera del país. El programa de construcción de carreteras y ferrocarriles de Pedro Nel Ospina en 1925 que permitió la interconexión de mercados nacionales. El establecimiento del IFI, en 1940, institución que promovió y construyó la siderúrgica Paz del Rió en 1948, entre 1953 y 1955, las inversiones en obras de infraestructura por parte del Estado.

Finalmente se resalta la importancia de la Ley 81 de 1960 por el impacto directo que tuvo sobre el desarrollo del sector industrial siderúrgico y metalmecánico que estableció exenciones tributarias por 10 años a industrias que fueron creadas o ampliadas sustancialmente en los años subsiguientes creando una nueva cadena productiva alrededor de la industria metalmecánica. (Poveda, 1979, p. 97-106).

Por otra parte, la opinion del sector privado con relación a la intervencion del Estado se puede percibir a traves del presidente de la Cámara de Fedemetal de la ANDI, quien en una entrevista realizada por la revista Metal Actual a la pregunta ¿Cuál es la posición del gobierno frente a la industria siderúrgica?, respondió que

El gobierno ha liderado y acompañado toda la creación de la agenda de competitividad sectorial que es una de las más completas que se ha concertado entre la industria y el gobierno y que más que el aporte de recursos económicos directos, se han desarrollado políticas sectoriales dirigidas a organizar aspectos como el suministro de materias primas, la infraestructura, los planes exportadores o la lucha contra el contrabando Adicionalmente, el Ministerio viene promoviendo programas como el desarrollo de proveedores a fin de fortalecer la relación proveedor empresa; aún debil en el sector siderurgico y metalmecánico del pais" (Metal Actual, 2008)

Esta opinion del representante gremial del sector siderúrgico en Colombia, deja ver en gran medida como la intervencion y acompañamiento del Estado siguen siendo aún prioridades para el desarrollo competitivo de la industria y por lo tanto para el establecimeinto de alternativas estrategicas de acción frente a un entorno que denota un alto grado de institucionalización nacional e internacional. 
A nivel sectorial, el gremio cuenta con las siguientes instituciones que muestran su cohesion interorganizacional para hacer presion en los cambios de las reglas de juego del entorno:

- Cámara Fedemetal dentro de la Asociación Nacionalde industriales, la cual cuenta con la afiliación de la totalidad de las siderurgias y las empresas importantes de laminación de la cadena en Colombia.

- Comité siderúrgico, que representa a la Secretaria Regional del Instituto Latinoamericano del Fierro y el Acero (ILAFA)

- Se desarrolla anualmente el Congreso Latinoamericano de Siderurgias

Esta dinámica interorganizacional gremial, también puede influir de algún modo en la profesionalización de prácticas de gestión que se institucionalizan en las empresas del sector, lo cual constituye una más de las formas de institucionalización al lado de las imposiciones estatales y las prácticas de estandarización por la presion competitiva del entorno. Aunque esto es sólo una hipótesis, sería un interesante tema que complemente la investigación desde el analisis institucional.

\subsection{El sector en Colombia}

En Colombia, los antecedentes de la industria siderúrgica, se remontan al siglo XIX con las experiencias de las siderúrgicas de Samacá (1856) en Boyacá, Pacho (1837) y la Pradera (1858) en Cundinamarca y Amagá en Antioquía (1865), empresas que no prosperaron debido a las serias dificultades técnicas y productivas que encontraron para el desarrollo de su actividad, en una época en que la experiencia industrial del país era incipiente y el mercado nacional muy reducido.

Ya entrado el siglo XX, la industria siderúrgica surge como resultado de las medidas proteccionistas del gobierno. Durante la primera presidencia (1934 -1938) de Alfonzo López Pumarejo, se promovió la sustitución de importaciones y el Estado expidió la Ley 97 de 1938 que autorizaba al gobierno a crear y financiar empresas siderúrgicas.

En 1940 el gobierno creó el Instituto de Fomento Industrial (IFI) que financió y participó en la creación de industrias en diversos renglones económicos. "En los sectores de sustitución tar- día, se destaca su participación en la creación de empresas siderúrgicas, automotrices, metalmecánicas y químicas" (Ocampo, 1994, p. 277).

Como resultado de las misiones geológicas emprendidas por el IFI, el Ingeniero Olimpo Gallo, descubrió las minas de hierro en el municipio de Paz del Río en 1941, y renacieron los intereses de los gobiernos Nacional y Departamental en la consolidación de un sector que ya desde la Revolución industrial se había denominado "Fábrica de Fábricas". Entonces, se creó en 1948, la Siderúrgica Nacional de Paz del Río, que cambió de razón social en 1954 y pasó a denominarse Acerías Paz del Río S.A.

Según la Encuesta Anual Manufacturera del DANE, para Colombia, el sector siderúrgico y metalmecánico representa aproximadamente el $12 \%$ del Producto Interno Bruto Industrial. Existen aproximadamente 1.200 establecimientos dentro de la cadena, los cuales generan más de 70.500 empleos.

En el Gráfico 1 se identifican los departamentos en los cuales se han concentrado las actividades siderúrgicas y se observa porqué Boyacá es un departamento importante en la ubicación de la industria siderúrgica del país, gracias a la generosa dotación de recursos minerales que posee.

Grafico 1. Producción de la industria siderurgica por departamentos

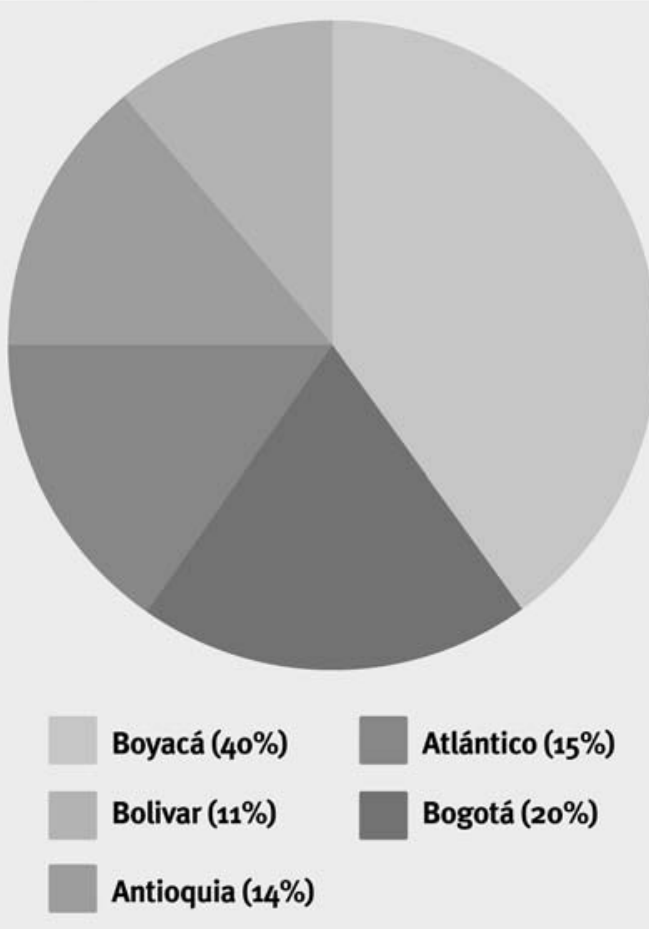

Fuente: Dane senso sectorial 2005. 
En Colombia, existe cierto grado de concentración del sector siderúrgico en varios grupos empresariales que han integrado pequeñas y medianas siderúrgicas para poder competir en los mercados nacionales y frente a las empresas internacionales que han incursionado en el país. Según el Documento sectorial, Metalmecánica y siderurgia del Departamento Nacional de Planea- ción (2007) en Colombia el sector siderúrgico presenta la siguiente composición:

El 65\% de las empresas del sector son pequeñas empresas con menos de 49 trabajadores y el $81.3 \%$ de la producción está concentrada en las grandes empresas que tienen más de 200 trabajadores (DNP, 2007). Las empresas siderúrgicas más grandes del país se encuentran identificadas en el cuadro 2.

\begin{tabular}{|c|c|c|}
\hline Año & Empresa & Lugar de ubicación \\
\hline 1934 & Siderúrgica Corradine & Pacho - Cundinamarca \\
\hline 1938 & Empresa Siderúrgica. Hoy Siderúrgica de Medellín S.A.- SIMESA & Medellín, Antioquia \\
\hline 1947 & Siderúrgica del Muña & Sibaté - Cundinamarca \\
\hline 1948 & $\begin{array}{l}\text { Siderúrgica Nacional de Paz del Río única siderúrgica integrada. } \\
\text { Denominada Acerías Paz de Río en } 1954 \text { Cuando fue inaugurada. }\end{array}$ & Belencito, Boyacá \\
\hline 1961 & Siderúrgica del Pacífico - SIDELPA & Cali, Valle del Cauca \\
\hline 1961 & Metalúrgica de Boyacá Hoy, Siderúrgica de Boyacá S.A. & Tuta, Boyacá \\
\hline 1965 & Siderúrgica del Norte - SIDUNOR & Barranquilla, Atlántico \\
\hline 1966 & Aceros Ramson hoy conocida como HORNASA. & Sogamoso, Boyacá \\
\hline 1970 & Acerías de Colombia S.A. - ASESCO & Pensilvania, Caldas \\
\hline 1972 & Distribuidora de Aceros Colombianos. DIACO Ltda & Medellín, Antioquia \\
\hline 1970 & Laminados Andino & Duitama, Boyacá \\
\hline 1979 & Aceros Boyacá & Paipa, Boyacá \\
\hline 1981 & Metales y procesos del Oriente MEPSA S.A. & Duitama, Boyacá \\
\hline 1981 & Siderúrgica del Caribe & Cartagena, Bolívar \\
\hline 1981 & Siderúrgica Nacional - SIDENAL S.A. & Sogamoso, Boyacá \\
\hline 1986 & Siderúrgica de Occidente - SIDOC & Cali \\
\hline 1992 & Siderúrgica de Caldas - S.A.S. ACASA. & Manizales \\
\hline
\end{tabular}

Fuente: elaboración propia con base en las páginas web de las empresas y fuentes.

Actualmente la industria ha afrontado distintos procesos de integración con empresas multinacionales que han modificado, tanto las acciones estratégicas individuales de las empresas como el papel del Estado en la dinámica del sector. El gráfico 2, con base en información de Proexport (2009), ilustra como las principales empresas siderúrgicas han venido realizando ventas, fusiones o adquisiciones tanto entre empresas colombianas como, con empresas internacionales.

Acerías Paz del Río es la única siderúrgica integrada del país, fundada en 1947 con $51 \%$ de acciones del gobierno nacional, y con sus propias minas de extracción de hierro, sin duda es un punto de referencia ineludible en la historia de la siderúrgica colombiana. Sin embargo desde el año 2008 la empresa fue adquirida por el grupo brasilero Votorantim, líder de la industria siderúrgica en dicho país (Gráfico 2)
Actualmente, entre los últimos movimientos de adquisiciones se identifica la compra de Laminados Andino ubicada en la ciudadela industrial de Duitama, por la empresa Diaco que pertenece al grupo Gerdau de origen brasilero.

Por otra parte en los elementos encontrados dentro de la agenda sectorial de competitividad del sector siderúrgico en Colombia, se encuentran claramente definidas acciones empresariales y acciones de intervención y regulación estatal.

Si bien el sector hizo solicitudes importantes, la acción que más solicitaron el sector y las regiones fue la de Instituciones para el desarrollo productivo. Dentro de este rubro se encuentran aspectos relativos a la administración pública, ajustes institucionales y diseño de políticas, el fortalecimiento institucional - fortalecimiento de la DIAN, Fomipyme y el Ministerio de Comercio, Industria y Turismoincentivos para las exportaciones, inversión en infraestructura para el sector, control de prácticas 


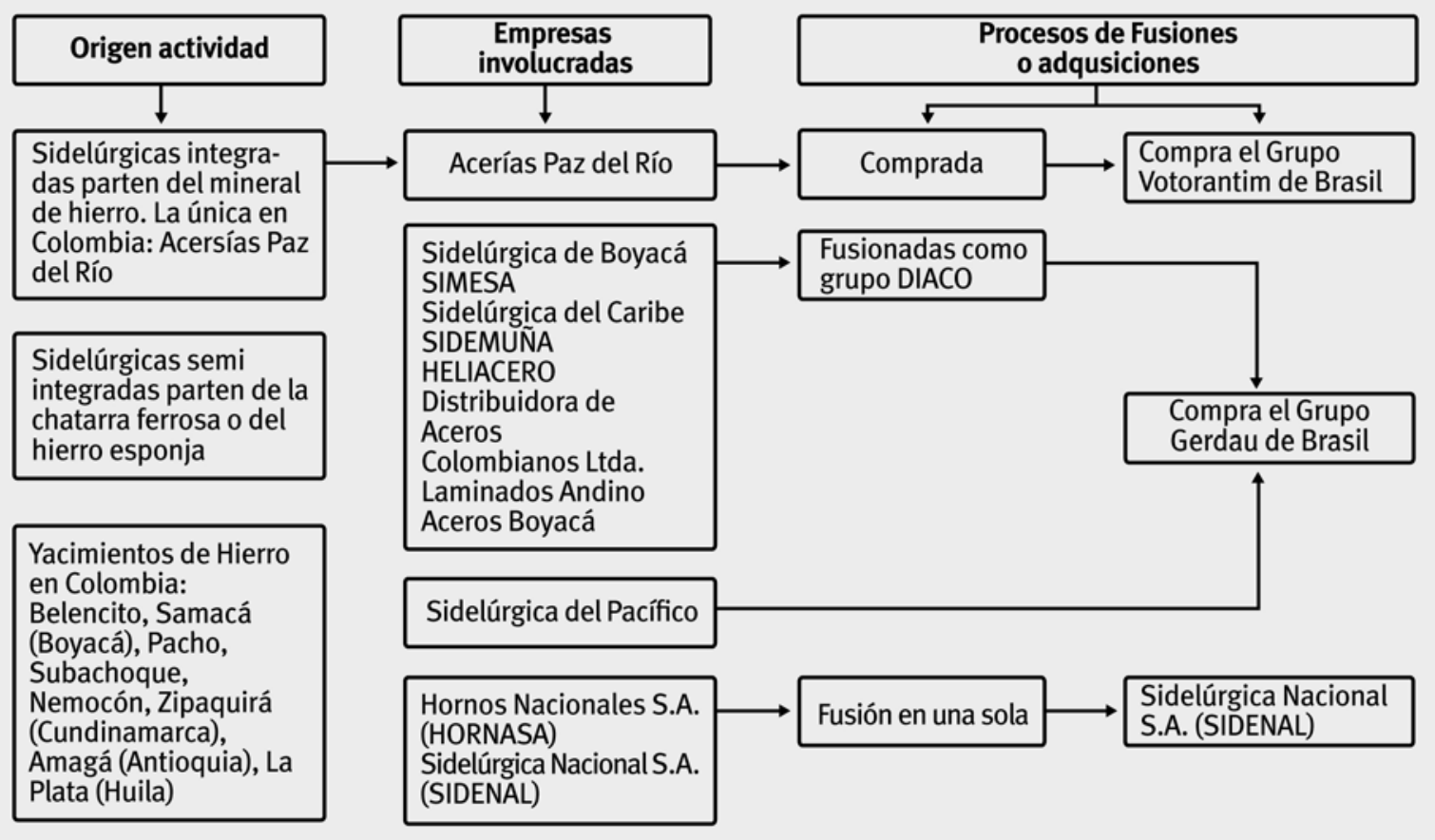

Fuente: elaboración propia con base en datos de Proexport 2009.

desleales e ilegales en el comercio internacional como dumping, subsidios, contrabando y salvaguardias. (Agenda Sectorial de competitividad. 2007).

Las propuestas por el sector buscan reformar el marco regulatorio, mejorar el acceso a materias primas, incrementar la competitividad del aparato logístico del sector, reducir tarifas de servicios públicos, implementar prácticas comerciales legales y leales y reducir trámites

\subsection{Ajustes regulatorios e instrumen- tos de apoyo para el sector siderúrgico en Colombia}

Según la Agenda Sectorial de Competitividad 2007, una de las grandes preocupaciones del sector son la claridad y la estabilidad del ambiente regulatorio. Para esto, la Ley 923 de 2005 estableció un marco y creó los contratos de estabilidad jurídica que les aseguran a los inversionistas por el término de duración del contrato la aplicación de las normas que hayan identificado como determinantes para realizar la inversión y que en caso de ser modificadas, podrían afectarlos negativamente.

El sector ya había manifestado su preocupación por asegurar el suministro de materias primas ante el Ministerio de Comercio, Industria y Comercio y estaba consagrado en los Convenios de Competitividad suscritos por el sector. Las limitaciones a las exportaciones de chatarra pueden ser discutidas en el Comité de Asuntos Aduaneros, Arancelarios y de Comercio Exterior (Comité Triple A), ya que esta decisión no depende exclusivamente del Ministerio de Comercio. En el 2019, el Gobierno espera conferir a las comisiones departamentales de Ciencia y Tecnología un papel primordial en el desarrollo de las agendas regionales de este sector. (Agenda Sectorial de competitividad. 2007)

Particularmente desde la creación del Ministerio del Medio Ambiente, Vivienda y Desarrollo Territorial se ha establecido un marco regulatorio relacionado en términos ambientales $^{1}$ que orienta tanto el diseño de

1. Resolución 909 de 2008 MAVDT sobre la Norma de Emisión admisible de contaminantes a la atmósfera por Fuentes Fijas. Política de residuos peligrosos (2005). Resolución 619 de 1997 MAVDT, sobre Industrias, obras, actividades o servicios que requieren permiso de emisiones. Resolución 898 de 1995. MAVDT sobre Certificado de Calidad de los combustibles líquidos. Decreto 948 de junio de 1995 MAVDT En relación con la prevención y control de la contaminación atmosférica y la protección de la calidad del aire. 
políticas, el establecimiento de planes, programas o proyectos y las bases para las sanciones jurídicas aplicadas a quienes generen daños ambientales, es decir, constituyen el marco de referencia para cualquier gestión en términos de responsabilidad ambiental del sector siderúrgico en el país ${ }^{2}$.

\subsection{Marco normativo del sector siderúrgico en Colombia}

En términos de la estandarización de procedimientos orientados al mejoramiento continuo y obtención de procesos de calidad para el sector siderúrgico se encuentra que las empresas se adscriben tanto a los procesos de certificación internacional ISO 14001 de procesos de gestión ambiental e ISO 9001 de certificación del sistema de gestión de la calidad, como a las Normas Técnicas de Calidad que el ICONTEC ha estipulado específicamente para certificar la calidad de los productos siderúrgicos elaborados en el país.

Las normas de calidad dan cuenta de los procesos de certificación que las empresas deben adelantar para garantizar que sus actividades productivas correspondan a unos estándares mínimos de cuidado ambiental a partir de los diseños de los productos y la infraestructura de producción disponible.

Adicionalmente, las normas presionan a las empresas para obtener la certificación y ajustarse a los protocolos que contribuyen tanto a controlar y minimizar los niveles de contaminación ambiental como a competir con los productos internacionales dentro y fuera del país.

De alguna manera, esta normatividad entendida como un requisito previo para las licitaciones con el Estado y para la incursión en mercados internacionales, constituye un factor externo de presión para el ajuste interno de los procesos de producción y gestión de las empresas sectorialmente, lo cual refleja el segundo nivel de institucionalización, analizado tanto por Hoffman como por Scott, citados en el marco conceptual, como una tendencia estratégica de isomorfismo.

\section{Reflexiones Finales y Conclusiones}

Dentro de los pilares del institucionalismo definidos por Scott, el grado de evolución que se evidencia en el sector siderúrgico ha avanzado de un término regulatorio que se inició con las políticas explícitas de protección y desarrollo industrial de finales del siglo XIX y las 4 primeras décadas del siglo $\mathrm{XX}$ a una dinámica normativa en la que las empresas del sector siderúrgico han entrado en la estandarización de procesos y de rutinas e incluso de las prácticas administrativas y estratégicas de integración.

Se identifica en el comportamiento de las empresas en los diferentes escenarios una tendencia inicialmente de conformidad, con las normas y la estructura regulatoria establecida por los respectivos gobiernos, que surge por la fuerte dependencia que la actividad siderúrgica tiene en relación con la infraestructura, la disponibilidad y el acceso a las materias primas que respondan a las necesidades del mercado, lo cual puede relacionarse con un mecanismo de isomorfismo dentro de la teoría institucionalista que puede ser característico del sector.

Se detecta un cambio en la respuesta estratégica de las organizaciones a partir del periodo de apertura económica, en el cual las presiones competitivas del mercado hacen que las organizaciones busquen nuevas alianzas estratégicas con empresas nacionales y extranjeras con el fin de mejorar su capacidad tecnológica, sus niveles de producción y su sostenibilidad en el mercado.

La intervención del Estado ha venido cambiando en la dinámica del entorno institucional del sector siderúrgico, pasando de un papel de creador, regulador o inversionista a un papel más mediador entre el Estado y el mercado.

2. NTC 1427, chatarra de fundición de hierro. Clasificación y características generales descriptores: chatarra; desechos de fundición de hierro; chatarra de fundición de hierro. NTC 1261, chatarra de acero. Clasificación y características generales descriptores: chatarra de acero. NTC 3353, Siderurgia definiciones y métodos para los ensayos mecánicos de productos de acero, NTC 2289 Barras corrugadas de acero de baja aleación para refuerzo de concreto, NTC 330, Para alambrón trefilable NTC 161 Barras y rollos lisos y corrugados de acero al carbono fuente: Ministerio de Minas y Energía e ICONTEC. 
Adicionalmente, existe una gran brecha entre la orientación de las acciones estratégicas asumidas por el sector siderúrgico de los países desarrollados y las planteadas desde la política sectorial en la industria siderúrgica colombiana. (Cuadro 3)

Cuadro 3. Apuestas competitivas internacionales en comparación con las estrategias propuestas por el sector siderúrgico en Colombia

Estrategias internacionales del sector siderúrgico

* Aceleración del proceso de consolidación sectorial

* Sector fragmentado

* Disciplina de mercado y estabilidad de precios

* Calidad y disponibilidad de los recursos humanos con los que realizar una gestión calificada y atender los procesos de I+D en todos los ámbitos de la actividad

* Deslocalización

* Especialización de productos y mercados

* Especialización de la gestión comercial y fidelización del cliente y la investigación para nuevos productos
Apuestas estrategicas nacionales del sector siderúrgico en Colombia

* Instituciones para el desarrollo productivo

* Reformar el marco regulatorio

* Mejorar el acceso a materias primas

* Incrementar la competitividad del aparato logístico del sector, reducir tarifas de servicios públicos

* Implementar prácticas comerciales legales y leales y reducir trámites

* Desarrollo empresarial: asociación empresarial, alianzas estratégicas y desarrollo del empresariado Fuente: elaboración propia con base en Datos de Proexport (2007).
El cuadro permite hacer dos inferencias, la primera es que en el escenario internacional, se están desarrollando acciones estratégicas centradas en las capacidades y recursos de las organizaciones, lo cual se explica por los procesos de apertura global del mercado siderúrgico y por la relativa estabilidad del apoyo proteccionista de sus respectivos gobiernos.

La segunda es que en el listado de las apuestas sectoriales del escenario siderúrgico colombiano, se evidencia una gran necesidad de intervención y apoyo del Estado para otorgar condiciones estructurales de crecimiento y competitividad del sector para poder desarrollar capacidades internas para competir, lo que refleja una desprotección del sector después del período de apertura de mercado.

Estas reflexiones apenas dejan planteada una parte del extenso análisis que implica abordar el estudio del comportamiento estratégico de un sector en el ambiente institucional. Quedan aún muchos campos por seguir analizando, tales como las relaciones entre las estructuras internas de las organizaciones y el desarrollo de sus capacidades y recursos con las disposiciones institucionales que caracterizan el sector, así como los grados de flexibilidad y autonomía estratégica que han identificado las empresas frente a las nuevas condiciones del mercado dentro de un marco institucional.

\section{Referencias}

Colombia, Ministerio de Ambiente, Vivienda y Desarrollo Territorial MAVT y - CECODES (2004). Construcción de una propuesta para la gestión ambiental sectorial orientada a las nuevas tendencias del desarrollo sostenible. Bogotá, Colombia: Ministerio de Ambiente, Vivienda y Desarrollo Territorial.

Colombia, Ministerio de Ambiente, Vivienda y Desarrollo Territorial MACT y CECODES. (2009). El ABC, del Cambio Climático en Colombia. En: http://www.minambiente.gov.co/ documentos/211209_abc_del_cambio_climatico_en_colombia.pdf.

Colombia. Ministerio de Comercio Industria y Turismo - Proexport. (2009). Invierta en Colombia. Trabajo, compromiso, ingenio. En el sector Siderúrgico. Consultado el 23/04/2010 de http://www.inviertaencolombia.com.co/Adjuntos/249_Sector\%20 Sider\%C3\%BArgico.pdf.

Deniz, H. (2007). La economía global y la industria siderúrgica. Boletín de Estudios Económicos LXII (192), pp. 445-454.

Departamento Nacional de Planeación. (2007). Documento sectorial, metalmecánica y siderúrgica. Agenda interna para la productividad y la competitividad. Bogotá, Colombia: DNP. 
Departamento Nacional de Planeación. (2010). La producción de 2002 y 2003 se estimó con base en EAM y muestra mensual manufacturera. En Siderurgia. Generalidades de la cadena productiva. http://www.dnp.gov.co/Portals/o/ archivos/documentos/DDE/Sidelurgia.pdf.

Díaz, M.P., Escudero, A. y Saenz, M.A. (2008). El desmantelamiento de la siderurgia integral del Mediterráneo Español (1977-1984). Revista de Historia Industrial, 17(38).

Giacalone, R. (2004). La regionalización del acero en América Latina: el caso del consorcio Amazonia. Buenos Aires, Argentina: Biblos.

Hoffman, J.A. (2001). De la herejía al dogma. Una historia institucional del ambientalismo empresarial. Palo Alto, CA: Stanford Bussines Books.

Montenegro, T.S. (2006). Sociedad abierta, geografía y desarrollo: ensayos de economía política, pp. 317. Bogotá, Colombia: Norma.

North, D. (1993). Instituciones, cambio institucional y desempeño económico. México D.F.: Fondo de Cultura Económica.

Oliver, C. y Holzinger, I. (2008). The effectiveness of strategic political management: and dynamic capabilities framework. Academy of Management Review, 33 (2), pp. 496-520.

Oliver, C. (1991). Strategic Reponses to Institutional Process. Academy of Management Review, n. 16, pp. $145^{-179}$.

Oliver, C. (1997). Sustainable Competitive Advantage Combining Institutional and resourcebased Views. Strategic Management Journal, 18(9), pp. 697-713.

Ospina, V.L. (1987). Industria y protección en Colombia 1810-1930. Medellín, Colombia: Biblioteca colombiana de ciencias sociales FAES.
Pfeffer, J. y Salancik, A. (1978). The Design and Management of Externally Controlled Organizations, en The External Control of Organizations cap 10, pp.257-287.Stanford, CA: Harper \& Row.

Porter, M.E. (1985). Estrategia competitiva: técnicas para el análisis de los sectores industriales y la competencia cap. 1, pp. 19-47 en Ventaja competitiva.

Porter, M.E. (1986). Contribuciones de la organización industrial para el management estratégico. Academy of Management Review, 6(4), pp. 609-620.

Poveda, R. (1979). Políticas Económicas, Desarrollo Industrial y Tecnología. En Colombia, 1925-1975 p. 156. Bogotá, Colombia: COLCIENCIAS.

Powell, W. y Dimaggio, P. (1999). El Nuevo institucionalismo en el análisis organizacional. México D.F.: Colegio nacional de ciencias políticas y administración pública, Universidad Autónoma del Estado de México y Fondo de Cultura Económica.

Ramírez, J. H. (1989). Una industria colombiana en el subdesarrollo: El desarrollo industrial frente a la reestructuración. Boletín cultural y bibliográfico, 1- XXVI (20). Recuperado el 23/04/2010 de http://www.banrepcultural. org/blaavirtual/publicacionesbanrep/boletin/boleti5/bol2o/resena9.htm.

Revista Metal Actual (2007) Entrevista a Juan Manuel Lesmes: "La cadena tiene claro su futuro y sabe hacia dónde va". n5, pp. 6-11. Recuperado el 15/03/2010 de http://www. metalactual.com/revista/5/entrevista.pdf.

Scott, R.W. (1995). Contemporary Institutional theory. In Institutions and organizations cap 3, pp. 33-61. California, EUA: Foundations for Organizational Science A Sage Publications series. 